CAHIERS DE

NARRATOLOGIE

\section{Cahiers de Narratologie}

Analyse et théorie narratives

31 Bis | 2017

Espace du récit, récit de l'espace en contexte germanique

\title{
« Ich mach ganz oft Kerzen an » ou comment raconter son habitat
}

\section{Britta Jallerat-Jabs}

\section{(2) OpenEdition}

Journals

Electronic version

URL: http://journals.openedition.org/narratologie/7668

DOI: 10.4000/narratologie.7668

ISSN: 1765-307X

Publisher

LIRCES

Electronic reference

Britta Jallerat-Jabs, « « Ich mach ganz oft Kerzen an » ou comment raconter son habitat », Cahiers de Narratologie [Online], 31 Bis | 2017, Online since 26 June 2017, connection on 22 April 2019. URL:

http://journals.openedition.org/narratologie/7668; DOI : 10.4000/narratologie.7668

This text was automatically generated on 22 April 2019

Article L.111-1 du Code de la propriété intellectuelle. 


\title{
« Ich mach ganz oft Kerzen an » ou comment raconter son habitat
}

\author{
Britta Jallerat-Jabs
}

\author{
Wohnen ist nicht nur ein basaler, mit dem \\ Menschen notwendig verbundener Prozess, es \\ «sucht » seine sprachliche Repräsentation [...]
}

1 Les lignes qui suivent se basent sur une enquête de terrain, menée par nos soins, en plusieurs étapes, entre 2005 et 2010. Nous avons réalisé une enquête qualitative, par entretiens semi-directifs, lors de laquelle nous avons rencontré 37 informateurs, locuteurs germanophones, dans plusieurs grandes villes d'Allemagne. Les interviews menées avec ces informateurs ont donné lieu à environs 37 heures de parole enregistrée, et près de mille pages de parole retranscrite. L'enquête portait sur le style de vie $^{2}$ des informateurs, que nous avons tenté de cerner en les interrogeant sur leurs pratiques de loisirs, sur leurs habitudes en matière de sociabilité, sur leurs goûts vestimentaires - et aussi sur leur habitat ${ }^{3}$.

2 Lors de ce type d'enquête qualitative, la situation de communication est particulière, différente de la communication spontanée de la vie de tous les jours. Ce qui distingue notamment la situation d'interview d'une conversation spontanée, c'est le caractère planifié de l'entretien (l'interview se déroule à une date, à une heure et dans un lieu convenus préalablement entre l'enquêteur et l'informateur, qui ont spécialement et uniquement rendez-vous dans le but de réaliser cet entretien), le cadre formel imposé (généralement, l'enquêteur exige que l'entretien se déroule en la seule présence de l'informateur ; l'entretien est enregistré), et le caractère asymétrique des rôles (les sujets sont imposés par l'enquêteur, les questions sont posées uniquement par l'enquêteur qui reste à tout moment un inconnu ne dévoilant rien de lui-même). Aussi, dans cette situation de communication inhabituelle, l'informateur cherche-t-il, comme le souligne Blanchet, à ajuster son discours à l'enquêteur, qu'il risque de percevoir comme un "représentant institutionnel du "savoir" » : on répond alors au chercheur « ce qu'on croit qu'il attend pour lui faire plaisir ou ce qu'on croit convenable de dire dans cette situation ${ }^{4}$ ». 
Ce qui est le plus souvent attendu des informateurs participant à ce type d'enquête qualitative, c'est qu'ils «racontent» (des épisodes de / des aspects de) leur vie, « raconter » étant compris ici au sens large proposé par Ehlich qui distingue, pour la langue allemande, deux acceptions ambivalentes du verbe "erzählen» (raconter): «erzählen ${ }_{1}$ " et «erzählen ${ }_{2}^{5}$ ». Le premier sens de raconter, appelé « raconter $_{1}$ » ("erzählen 1 »), recouvre en tant que terme générique des activités langagières aussi variées que "rapporter", "décrire", "restituer", " exposer", et englobe aussi « raconter $_{2}$ " (« erzählen $\left.{ }_{2} »\right)$, correspondant à ce que l'on entend généralement par « raconter une histoire ». Ehlich illustre la différence entre les deux acceptions du verbe «erzählen» de la façon suivante : "Quand on demande à quelqu'un de raconter ses vacances, il peut répondre tout aussi bien par un bref rapport ou un simple exposé du déroulement des vacances ( raconter $_{1} »$ ) que par une série de petites histoires ( $\left(\text { raconter }_{2} »\right)^{6} »$. À l'occasion d'une interview, la forme précise que prendra le discours (c'est-à-dire une des formes de " raconter $_{1}$ » ou la forme " raconter $_{2}$ ") dépend donc non seulement des questions posées par l'enquêteur et des sujets abordés, mais aussi des choix discursifs des informateurs.

4 Lorsque ces derniers optent pour la variante « raconter $_{2}$ ", il faut cependant tenir compte d'une autre spécificité générée par la situation d'enquête. En effet, comme le précisent Heinemann et Viehweger, quand on raconte une histoire dans la vie de tous les jours, « les actions que l'on raconte doivent être intéressantes pour l'allocuté, et dans la plupart des cas, cet intérêt est suscité par un décalage par rapport aux normes ou par le fait que les attentes liées au déroulement normal d'une action ne sont pas satisfaites ${ }^{7}$ ». Or, Rehbein fait remarquer à juste titre que, lors de la "narration biographique " (" biographisches Erzählen »), les informateurs racontent des histoires non pas forcément pour leur caractère exceptionnel et inattendu, ou parce qu'il s'agit d'expériences particulièrement intéressantes, mais aussi dans le but d'exemplifier, d'illustrer l'habituel : " on raconte comment "cela se passe d'habitude" " ". Ceci s'explique très facilement par le fait que le chercheur qui réalise une enquête de terrain ne cherche généralement pas l'exceptionnel, mais le régulier, les règles, les normes qui déterminent et expliquent le comportement humain. Par ailleurs, il est à noter que, dans les récits biographiques (comme dans beaucoup d'autres situations de communication), on est le plus souvent confronté à un emboîtement ("Verschachtelung ») de différentes formes discursives?.

Revenons à présent à la spécificité thématique de notre enquête: le style de vie de nos informateurs. Auer pense que «souvent, les membres d'une communauté culturelle et linguistique sont conscients de l'existence de styles qu'ils savent même en partie désigner. Le style est quelque chose dont il est possible de parler ${ }^{10}$.» Il est vrai que le terme de "style» a massivement envahi le langage de tous les jours, et s'applique désormais à quasiment tous les secteurs de la vie quotidienne. Manifestement, Auer fait ici allusion à cet emploi vulgarisé du terme. Il fait référence, dans le passage que nous venons de citer, au phénomène du style de façon générale; or, ce qui nous intéressera plus particulièrement ici, c'est le style d'intérieur (Wohnstil). Lors de notre enquête, nous avons en effet tenté de vérifier l'affirmation de Auer dans un des domaines où le style (pris dans ce sens vulgarisé) se déploie : l'habitat. Auer utilise deux termes évoquant des activités langagières: "désigner» («benennen») et, de façon beaucoup moins précise, « parler de » (« sprechen über »). 


\section{Désigner}

6 Il est vrai que l'allemand contemporain, se servant volontiers de sa facilité à composer des lexèmes nominaux ad-hoc, dispose de termes permettant de désigner des styles d'intérieur : Bohème-Stil, Ethno-Stil, Industrial-Stil, Retro-Stil, Vintage-Stil, pour ne citer que quelques exemples que l'on trouve dans des revues ou sur des sites Internet spécialisés dans la décoration d'intérieur. Cependant, lors des interviews, il s'avère que nos informateurs ne connaissent pas ces termes, ou, tout du moins, ne les mobilisent pas : la question de désigner ( «bezeichnen ») leur style d'intérieur semble les mettre dans un certain embarras, comme le montrent les extraits d'entretien ci-dessous :

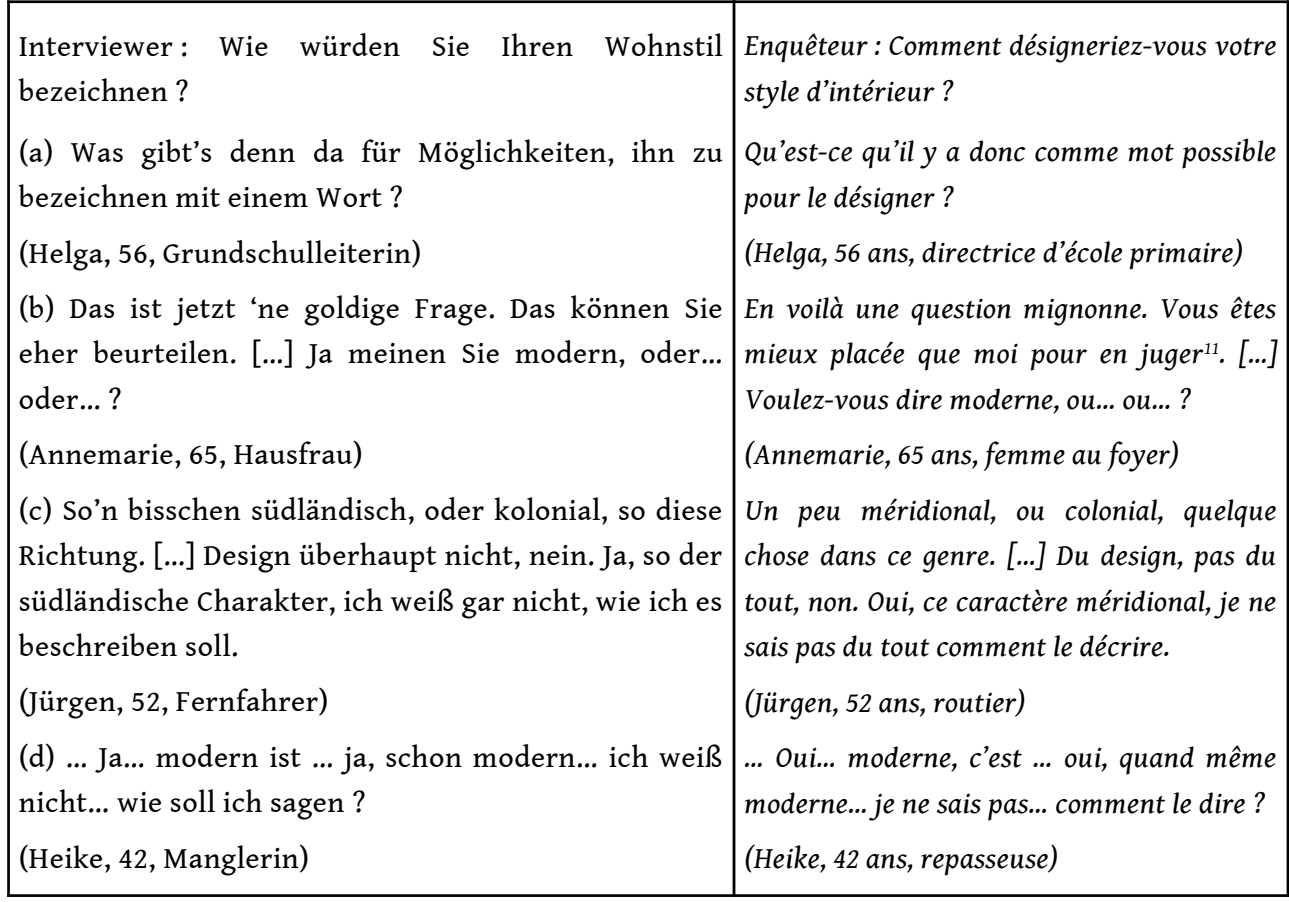

7 Tandis que Helga (a) se déclare incompétente simplement sur le plan lexical, Annemarie (b) semble surprise qu'on lui demande de désigner son propre style, comme si cela ne pouvait pas revenir au "porteur» d'un style d'apprécier ce dernier. Jürgen et Heike expriment explicitement qu'ils ne trouvent pas les mots adéquats pour répondre à la question. Les réactions reproduites sous (a) et (b) sont par ailleurs remarquables parce que tout à fait inhabituelles dans une situation d'interview, dans la mesure où les informateurs s'adressent directement à l'enquêteur (en l'occurrence nous-même) en sollicitant de l'aide (a), voire en lui demandant de répondre à leur place (b). Ceci ne correspond pas aux rôles tels qu'ils sont habituellement distribués lors de l'interaction que représente une interview, où l'enquêteur n'est pas un interlocuteur à qui l'on pose des questions, avec qui l'on cherche à établir un dialogue, mais une sorte d'instance à laquelle il revient d'interroger ${ }^{12}$.

8 Il se peut que nos informateurs ne connaissent pas ou n'emploient pas les termes permettant de désigner des styles d'intérieur parce que ce lexique reste du domaine d'un discours spécialisé13. Il est possible aussi que les types d'intérieurs désignés par ces termes - dans la presse, dans les médias spécialisés - correspondent à des idéaux qui 
n'existent que dans les magasins ou les magazines d'ameublement et de décoration. Mais il est également envisageable que les informateurs soient désorientés par le fait que l'enquêteur leur demande de désigner. Désigner suppose la connaissance du «terme juste », et c'est bien ce terme qui semble faire défaut à nos informateurs, comme le montrent les extraits d'entretien plus haut $(\mathrm{a}-\mathrm{d})$. On propose maintenant de voir, à partir des exemples $(e-i)$ que les locuteurs, à défaut de savoir le désigner, s'efforcent de décrire leur style d'intérieur.

\section{Décrire}

9 Précisons au préalable que, même si nos informateurs produisent un discours descriptif sur leur espace d'habitation, il ne s'agit pas d'une description (« Beschreibung ») telle que l'entend par exemple Rehbein. Certes, Rehbein associe l'activité langagière de description prioritairement à des réalités spatiales, car il considère que «la description relève du domaine pragmatico-fonctionnel de l'orientation spatiale » (l'auteur souligne) ${ }^{14}$, et qu' " à l'occasion d'une description, l'allocuté "se déplace dans l'espace représenté15" ». Par conséquent, le groupe prépositionnel se trouve parmi les moyens linguistiques privilégiés lors de ces descriptions, dans la mesure où il s'agit d'un mouvement (imaginaire) dans l'espace et d'un positionnement des éléments les uns par rapport aux autres: « La direction et les étapes du déplacement font surgir dans la représentation une relation entre les différents éléments » (l'auteur souligne) ${ }^{16}$.

Or, pour les informateurs, interrogés par l'enquêteur sur leur style d'intérieur, il n'est pas question de livrer une description précise de l'agencement, donc de la réalité spatiale au sens étroit du terme ${ }^{17}$, de leur espace-logement, mais d'une impression générale que cet espace dégage, d'une ambiance; en conséquence, les moyens linguistiques déployés ne sont pas prioritairement des groupes prépositionnels, mais des groupes adjectivaux : il ne s'agit pas de situer, mais de qualifier. Quelques substantifs interviennent également, afin de mentionner des objets d'ameublement, de décoration ou des matériaux : 
(e) Ja, äh, ja auch wieder [Lachen] es ist komisch eigentlich, auch wieder in der Mitte. Also, nicht so, ich mag das nicht so sehr elegant oder so was, also dieses Brokat oder sonst was, überhaupt nicht. Aber natürlich auch nicht zu deftig.

(Mechthild, 65, im Ruhestand)

(f) $\mathrm{H}: \mathrm{Ja}$.... also... schon modern auf der einen Seite aber nicht, nicht... wiederum gemütlich soll's sein und individuell eingerichtet, also wir haben noch nie, oder ich habe noch nie Wohnwände gemocht oder Garnituren gemocht oder was immer so was ist, sondern wir haben Regale gemacht, gebaut und bauen lassen, wo's, wo Regale sein sollten, und Schränke, ja da haben wir dann eben die einzelnen Teile gekauft.

$\mathrm{I}^{18}$ : Also modern haben Sie gesagt, individuell und gemütlich.

$\mathrm{H}: \mathrm{Ja}$. Nix Geschnörkeltes und nichts Verspieltes so in dem Sinn...

(Helga)

(g) Behaglich. Gemütlich. [...] Dekoriert, nicht gerade Glas und Bausteine, und kühl und kalt, und nur modernes und strenges Design, sondern, ja, weicher, gemütlicher, ausdekoriert oder geschmückt oder gestaltet.

(Annemarie)

(h) Ich mixe auch mit Alt und Neu. Modern. Hab sehr moderne Bilder. Hab einen Jugendstilschrank. Meine Couch ist weiß. Leder. Kauf mir auch wieder 'ne weiße. Die Holzwand war mal dunkel, die hab ich weiß gestrichen mal, vor ein paar Jahren. Was hab ich ? Schöne bunte Kissen. Ähm, zwei Teppiche in blau-beige-Tönen... [...] Muss mich wohlfühlen, ja. Also meine Wohnung ist... hat keinen kühlen Touch. So von der Einrichtung her auch. Oder von den Farbklecksen. Wir haben das eben dann versucht, auch im Schlafzimmer, ein bisschen Wärme reinzubringen: weiße Wände, weißer Schrank, dann noch ein ziemlich heller Boden, da ist mir dann so 'ne Bananenklappkonsole (?) begegnet... Das sieht klasse aus mit dem weißen Boden. Ja. Und hab versucht auch dann noch "ne schöne Palme, ja? und alles in diesen warmen Tönen ein bisschen $\mathrm{zu}$ halten. Jetzt ist... ein Bild fehlt noch. Aber das ist mir noch nicht begegnet, cabiersidesentatalfigie, 31 Bis | 2017

(Renate, 58, Unternehmensleiterin)

(i) I: Und was würden Sie sagen, was für 'nen Wohnstil haben Sie? Wie...
Oui, euh, oui, là aussi à nouveau [rires], au fond c'est bizarre, là aussi quelque chose d'intermédiaire. Donc, pas tellement, je n'aime pas quand c'est genre très élégant, donc $d u$ brocart ou quelque chose comme ça, pas du tout. Mais évidemment pas non plus trop rustique.

(Mechthild, 65 ans, retraitée)

H : Oui. ... alors... certes, moderne, d'un côté, mais pas, pas... il faut en revanche que ce soit chaleureux, et meublé de façon personnelle, donc, nous n'avons encore jamais, ou moi je n'ai jamais aimé les livings ou les ensembles canapé-fauteuils, ou ce genre de chose, au contraire, nous avons fabriqué des bibliothèques, fabriqué ou fait fabriquer, là où il nous fallait des bibliothèques, et quant aux armoires, et bien, là, nous les avons achetées séparément.

$E^{19}$ : Vous avez donc dit donc moderne, personnel et chaleureux.

H: Oui. Je veux dire rien de tarabiscoté, pas de fioritures.

(Helga)

Confortable. Chaleureux. [...] Décoré, pas forcément du verre et de la pierre, et sobre et froid, et seulement du design moderne et austère, mais au contraire, plus douillet, plus chaleureux, très décoré ou ornementé ou bien arrangé.

(Annemarie)

Je mélange aussi l'ancien et le nouveau. Moderne. J'ai des tableaux très modernes. J'ai une armoire style art nouveau. Mon canapé est blanc. En cuir. Mon prochain sera blanc aussi. Le living était en bois foncé, je l'ai peint en blanc il y a quelques années. Quoi d'autre? De beaux coussins multicolores. Euh, deux tapis dans des tons bleu et beige... [...]. J'ai besoin de me sentir bien, oui. Alors, mon appartement n'est pas... ce n'est pas une ambiance froide. En ce qui concerne les meubles aussi. Ou les touches de couleur. Nous avons donc essayé, dans la chambre à coucher aussi, d'apporter un peu de chaleur : des murs blancs, une armoire blanche, un sol assez clair, et puis je suis tombée sur une console en bananier (?) ... Ça rend super bien avec le sol blanc. Oui. Et puis, j'ai essayé, avec un beau palmier, vous voyez? et de rester un peu dans ces tons chauds pour tout l'ensemble. Là... il manque encore un tableau. Mais je ne suis pas encore tombée dessus, mais ça arrivera un jour.

(Renate, 58 ans, chef d'entreprise) 
11 Avant de nous pencher plus en détail sur l'analyse des groupes adjectivaux, notons, dans un premier temps, que les cinq informatrices parlent de leur style d'intérieur en le comparant à un autre style qui lui est opposé, même si l'importance que prend cette confrontation varie selon la locutrice. Ainsi, Helga oppose le trait « modern » (moderne) à «Geschnörkeltes », «Verspieltes » (tarabiscoté, fioritures) et le trait «individuell » (personnel) à « Wohnwände » (livings), « Garnituren » (ensembles canapé-fauteuils). Elle explicite ce qu'elle entend par « individuell » : les bibliothèques, par exemple, sont faites sur mesure voire fabriquées par Helga et son mari, contrairement donc à des «Wohnwände » ou des «Garnituren » qui correspondent à du mobilier préfabriqué. Annemarie, quant à elle, déploie un vocabulaire abondant autour d'un seul trait: sept adjectifs qualifient le côté "décoré-chaleureux" de sa maison, dont l'ambiance est opposée à un intérieur sobre et froid.

12 On observe en effet que les informateurs ne se contentent souvent pas de décrire l'ambiance de leur propre intérieur: ils se distancient également d'autres styles d'intérieur, qui représentent l'antithèse de ce qui leur plaît, le contraire de ce qu'ils recherchent par leur propre façon de meubler et d'aménager leur espace-logement. Ceci nous rappelle qu'un style est un phénomène relationnel : il ne peut exister que dans la comparaison, dans l'opposition par rapport à d'autres styles ${ }^{20}$. Manifestement, ce trait définitoire de ce qu'est un style persiste dans l'emploi vulgarisé du terme.

13 Cette façon de procéder à des sortes de «démonstrations par la négative » dévoile une difficulté à laquelle sont confrontés les informateurs lorsqu'on leur demande de parler de leur appartement ou de leur maison. Nos informateurs se trouvent, en tant que participants à une enquête qualitative, dans une situation de représentation où il s'agit de se mettre en scène dans le but d'une valorisation de soi. La catégorie du style s'avère, dans cette perspective, bien plus contraignante que les catégories sociales traditionnelles: s'il est possible de parler d'un milieu d'origine modeste, d'un faible niveau d'étude, d'une situation professionnelle ou économique défavorables comme d'une fatalité ou d'une injustice sociale, le style est par définition le résultat de choix dont on est le seul responsable : il n'est pas envisageable de dire, ni même de penser, « ma maison n'est pas belle parce que j'ai mauvais goût ». En même temps, les locuteurs sont exposés à la « règle des fleurs » : c'est ainsi que Kerbrat-Orecchioni appelle le fait que les règles de bienséance dans notre société interdisent que l'on exprime, ouvertement, toute flatterie envers soi :

Il ne convient pas [...] de se glorifier soi-même. [...]

Règle de modestie, ou encore : règle qui interdit que l'on se jette ostensiblement des

fleurs à soi-même (p. abrév. : règle des fleurs) [...]

Cette règle est solidement inscrite, dans notre culture du moins ${ }^{21}$.

Devant ces exigences incompatibles - se mettre en valeur mais ne pas dire du bien de soi les locuteurs, interrogés sur le style de leurs intérieurs, trouvent des astuces discursives. Une de ces astuces semble résider dans le dénigrement de ce qui est contraire à soimême: quand nos informateurs décrivent leur style d'intérieur en disant à quoi il ne correspond pas, cet autre style (celui qui est différent voire l'opposé du sien) est, en passant, dévalorisé. C'est pourquoi nos informatrices adeptes, par exemple, d'un style de décoration qu'elles qualifient de " gemütlich» (confortable, douillet), opposent à celui-ci des qualificatifs comme « kühl » (sobre), « kalt» (froid) voire " nackt » (nu), évoquant respectivement le mal-être psychologique ou physique et la privation. 
De façon schématique, la description des ambiances semble s'organiser autour des pôles « chaud, chaleureux » (approuvé) et «froid» (désapprouvé); en témoignent les termes " gemütlich» (confortable), «behaglich» (douillet), « weich » (doux), "warm » (chaud), «Wärme » (chaleur) d'un côté, et « kühl » (sobre) ou « kalt » (froid), «streng » (austère), «nackt» (nu) de l'autre. "Modern» semble être un qualificatif qui divise : il apparaît aussi bien dans la rubrique des "styles approuvés» (Helga) que dans celle des «styles désapprouvés " (Annemarie). Le terme allemand "gemütlich» - réputé intraduisible en français et rendu ici de façon approximative par " confortable, douillet, chaleureux » bénéficie d'un emploi transversal ; apparemment, « gemütlich » est compatible aussi bien avec « modern » (" moderne », voir Helga) qu'avec « ausdekoriert » (" très décoré », voir Annemarie) et " einfach » (" simple », voir Meike). "Gemütlich » sert plus à décrire un sentiment (lié à un lieu où l'on se sent bien et à une situation dans laquelle on est à l'aise) que l'aspect visuel d'un intérieur. De façon encore plus manifeste, «schön » («beau », voir Renate) et «normal » («normal », voir Meike) traduisent des jugements subjectifs, à défaut de contribuer à une description concrète d'un espace. Les informateurs livrent également des informations plus objectives, par le biais d'adjectifs désignant ou qualifiant des couleurs, comme « weiß» (blanc) ou « hell » (clair), ou encore par certains substantifs désignant des matériaux (par exemple "Glas»=verre), des finitions (par exemple «Brokat» = brocart) ou des pièces de mobilier (par exemple "Jugendstilschrank » $=$ armoire style art nouveau).

\section{Raconter}

On note par ailleurs que chez certains informateurs, invités à parler de leur style d'intérieur, surgissent, à côté des éléments descriptifs, des amorces narratives, car ils racontent de quelle façon ils s'investissent activement dans la décoration et l'aménagement: « Regale bauen » (fabriquer des bibliothèques) ( $\mathrm{f}$ ), « Alt und Neu mixen » (mélanger l'ancien et le nouveau) (h), « weiß streichen » (peindre en blanc) (h), « Fenster schmücken » (décorer les fenêtres) (i), «Glastische dekorieren » (décorer des tables en verre) (i). Ce qui est mis en avant ici, ce sont donc des actions et, d'après Heinemann et Viehweger, c'est justement ce qui caractérise la narration: «Une des caractéristiques fondamentales de la narration conversationnelle consiste dans le fait que les contenus racontés concernent des actions accomplies par les individus ${ }^{22}$.» Il est vrai que le substantif composé "Wohnstil» par lequel nous visions a priori (pour les besoins de notre enquête portant - nous le rappelons - sur le style de vie) le style d'ameublement et de décoration, c'est-à-dire une image statique de l'aspect visuel de l'appartement («Stil der Wohnung / der Wohnungseinrichtung» =le style de l'appartement/ de l'ameublement), peut après tout connaître une lecture dynamique: en effet, le déterminant «Wohn-» de ce composé peut être compris comme «Stil des Wohnens » (le style / la façon d'habiter). C'est précisément dans ce sens que s'oriente la réponse de Charlotte : 


\begin{tabular}{|l|l|}
\hline (j) Also es muss nichts Designermäßiges sein, aber & Pas nécessairement du design en fait, mais il \\
es muss, ich muss mich total wohlfühlen, ich mag & faut que ce soit, j'ai besoin de me sentir \\
zum Beispiel nicht ein ungemachtes Bett. Und ich, & vraiment bien, je n'aime pas par exemple un lit \\
wenn ich aufstehe, und in so'n Kissen gequetscht & défait. Et quand je me lève, et que j'ai écrasé un \\
hab, dann mach ich einmal so: [richtet ein & coussin, je fais juste comme ça: [redresse un \\
Sofakissen auf]. Und Jörgi [= mein Mann] macht's & coussin du canapé]. Alors que Jörgi [mon mari] \\
halt nicht. Aber egal. Und ich falt auch meine & ne le fait pas. Mais passons. Et je replie aussi \\
Zeitung wieder zusammen und ich räum abends & mon journal, et le soir, je range le coin du salon \\
ihre Spielecke [= die Spielecke meiner Tochter] & où elle [= ma fille] a joué, avant de m'asseoir ici \\
auf, bevor ich mich hier hinsetze mit 'nem Buch, & pour lire un livre, car j'aime bien que tout soit \\
weil ich mich gerne umguck und es ordentlich & bien rangé quand je regarde autour de moi. Et \\
habe. Und wir kaufen auch jeden Samstag auf'm & tous les samedis, nous achetons de belles fleurs \\
Markt schöne Blumen. Und... die waren jetzt & au marché. Et... là, elles étaient fânées, \\
leider verblüht, sonst hättst du sie noch gesehen... & malheureusement, sinon tu les auraient encore \\
Und ähm, ja mir ist schönes Licht total wichtig, & vues... Et, euh, ce qui m'importe beaucoup, c'est \\
ich mach ganz oft Kerzen an, das ist mir schon & un bel éclairage, j'allume très souvent des \\
total wichtig. & bougies, c'est super important pour moi. \\
(Charlotte, 30, Grundschullehrerin) & (Charlotte, 30 ans, institutrice)
\end{tabular}
décoration, nous raconte ce qu'elle fait pour rendre l'activité "Wohnen" (habiter) agréable : maintenir l'appartement en ordre, acheter régulièrement des fleurs, allumer fréquemment des bougies. Nous apprenons, au passage, quelles activités elle a l'habitude d'exercer chez elle (lire le journal, lire des livres), et nous apercevons à travers son récit que le rapport à l'espace commun que constitue leur appartement est une source de conflit entre elle et son mari. Nous sommes désormais bien loin de l'activité langagière « bezeichnen » (désigner) - rappelons que c'était pourtant ce que nous avions demandé à nos informateurs. Ce que fait Charlotte correspond bien plus à nous raconter (" erzählen ») sa façon d'agir dans son espace-logement. Nous avons vu plus haut qu'une spécificité de la narration biographique était le récit du quotidien, de l'habituel (« erzählen, wie es immer ist»). Aussi trouvons-nous dans le discours de Charlotte plusieurs marqueurs d'itérativité : «wenn » («lorsque, quand», conjoncteur impliquant, tel qu'il est employé ici, l'idée de répétition: à chaque fois que), "jeden Samstag" (chaque samedi), « ganz oft » (très souvent), « gerne » (volontiers).

De façon similaire, Horst, après une brève et vague description du mobilier et des tableaux présents dans son appartement, en arrive rapidement à ce qui est pour lui le plus important: son logement doit être équipé de tout ce qui lui permet d'exercer ses activités préférées (les vidéos, les photos), de tout ce que rend la vie agréable, ce dont on peut profiter ("genießen »). Chez Horst, le récit se concentre également sur le point de vue dynamique de ce que l'on fait dans son appartement, plutôt que sur l'aspect visuel et statique : 
(k) Wohnstil, ja, nicht unbedingt mit Eiche, eher Style d'intérieur, oui, pas nécessairement des modern. Bilder, die müssen aber nicht meubles en chêne, plutôt moderne. Des tableaux, unbedingt echt sein, sie müssen nur gefallen, sie mais pas forcément des originaux, il faut juste müssen viel Farbe hergeben. Medien müssen qu'ils nous plaisent, qu'ils soient très colorés. Tous immer alle vorhanden sein, moderner les médias doivent être présents, une télé Fernseher, ein Digitalrekorder, dann moderne, un magnétoscope numérique, et puis fotografiere ich auch sehr gerne, filme gerne j'aime beaucoup prendre des photos, j'aime faire und bearbeite das alles [...] Ja, eigentlich ein des vidéos, et travailler dessus [...] Oui, en fait, un komfortabler Stil, so würde ich das bezeichnen, style confortable, c'est comme ça que je dirais, alles was man so genießen kann.

tout ce dont on peut profiter.

(Horst, 65, Schulleiter im Ruhestand)

(Horst, 65 ans, directeur d'école, retraité)

19 Un certain nombre de nos informateurs semblent donc plus vivre, et moins voir, leur espace-logement, ce qui peut expliquer cette tendance à introduire des éléments narratifs dans le discours. C'est ce qui explique peut-être aussi que des qualificatifs comme, par exemple, « gemütlich » ou « modern » puissent s'appliquer à des intérieurs très différents quant à leur aspect visuel.

\section{Les adjectifs}

Revenons un instant sur les adjectifs qualificatifs qui nous intéressent plus particulièrement, car dans notre corpus ce sont ces derniers, nous l'avons vu, qui interviennent prioritairement afin de décrire le style des intérieurs. La liste ci-dessous énumère (par ordre alphabétique) les adjectifs repérés dans les réponses de nos informateurs, en sachant que certains parmi ces adjectifs ne sont pas employés afin de décrire son style d'intérieur positivement, mais uniquement pour émettre des jugements négatifs (« deftig », « kalt », « kühl », « streng ») :

\begin{tabular}{|l|l|}
\hline $\begin{array}{l}\text { ausdekoriert, behaglich, chaotisch, deftig, } \\
\text { dekoriert, edel, einfach, elegant, gemütlich, } \\
\text { geschmückt, gestaltet, hübsch, individuell, kalt, } \\
\text { kolonial, kühl, modern, normal, praktisch, streng, } \\
\text { südländisch, verschnörkelt, verspielt, weich }\end{array}$ & $\begin{array}{l}\text { distingué, simple, élégant, confortable, } \\
\text { ornementé, arrangé, joli, personnel, froid, } \\
\text { colonial, sobre, moderne, normal, pratique, } \\
\text { austère, méridional, tarabiscoté, avec des } \\
\text { fioritures, doux }\end{array}$ \\
\hline
\end{tabular}

21 Nous constatons qu'un certain nombre de ces adjectifs sont employés dans un effort de description de l'aspect visuel : «elegant» (élégant), «modern » (moderne), «streng » (austère). D'autres visent prioritairement la caractérisation d'une ambiance ressentie : « behaglich» (douillet), « gemütlich» (confortable), «kalt» (froid), « kühl» (sobre). «Praktisch» (pratique) sélectionne le trait de la fonctionnalité. Ceux-là, même s'ils ne mettent pas le focus sur l'aspect visuel ou esthétique, possèdent néanmoins une certaine force évocatrice. L'allocuté arrivera certainement à se représenter un intérieur " élégant », «chaleureux ", «froid » etc. - même si ses propres représentations risquent d'être en décalage avec l'espace dont l'informateur est en train de parler (son logement). En revanche, «hübsch» (joli), «normal» (normal) et «individuell» (personnel) ne 
communiquent qu'une appréciation subjective sans laisser à l'allocuté la possibilité de se référer à des représentations disponibles dans le "stock social des connaissances" (d'après l'expression de Berger et Luckmann ${ }^{23}$ ), afin d'imaginer visuellement l'intérieur dont il est question.

Nous traitons les adjectifs de couleur (désignant ou caractérisant les couleurs) à part, car ce groupe se trouve être proportionnellement le plus important. La couleur, quand il est question de parler de son intérieur, semble donc correspondre à un paramètre qui mérite notre attention.

beige, blau-beige-Töne, bunt, cappuccino- beige, tons bleu et beige, multicolore, couleur farben, Gelbtöne (weiche), hellbraun, knallrot, cappuccino, tons jaune (doux), marron clair, rouge Maisfarbe, rot, Vanilla, weiß criard, jaune maïs, rouge, vanille, blanc

Lorsque nos informateurs commentent le choix des couleurs, il apparaît que les critères évoqués se situent plus dans le domaine symbolique qu'esthétique :

\begin{tabular}{|c|c|}
\hline $\begin{array}{l}\text { (l) Ja ich, als ich die Wohnung eingerichtet hab, } \\
\text { hatt ich natürlich auch viele Leute, die mir da } \\
\text { Tipps gegeben haben oder... und ich hab sehr viele } \\
\text { umstrittene Entscheidungen getroffen, ich hab } \\
\text { anderthalb Wände rot gestrichen, also knallrot. } \\
\text { (Joschi, 41, Informatiker) }\end{array}$ & $\begin{array}{l}\text { Oui je, quand j'ai fait la déco de l'appartement, } \\
\text { j'avais bien sûr un tas de gens qui m'ont donné } \\
\text { des conseils ou... et j'ai pris beaucoup de } \\
\text { décisions contestées, j'ai peint en rouge un mur } \\
\text { et demi, je veux dire en rouge criard. } \\
\text { (Joschi, } 41 \text { ans, informaticien) }\end{array}$ \\
\hline
\end{tabular}

Peindre des murs en rouge vif signifie pour Joschi faire preuve d'un style non conformiste, car il l'a fait en dépit des conseils de son entourage. Le rouge, couleur chargée symboliquement, est entre autres une couleur contestataire.

En revanche, les tons pastel, clairs, discrets semblent correspondre à un style qui cherche l'approbation sociale, la valorisation de la part d'autrui. Ce sont des tons que Gabi a choisis pour l'intérieur de son appartement en souhaitant l'aménager dans un style quelle qualifie de « edel » (distingué) :

(m) I: Wie würden Sie [Ihren Wohnungsstil] beschreiben?

G : Edel !

I : Aha, hmm. Und welches ist die Grundfarbe?

$G$ : Beige ! Beige, weiß. Also das ist nicht beige, das ist so mehr so "ne Maisfarbe, Vanilla, so ganz weiche Gelbtöne. Mit weiß. Und weißlackierte Schränke mit Holzplatten, so mit Maserung drauf. So ganz edel alles. Und dann so cappuccinofarben. (Gabi, 51, Kosmetikerin)
E: Comment décrieriez-vous votre décoration d'intérieur?

G : Distinguée!

E: D'accord. Et quelle est la couleur de base? $G$ : Beige! Du beige, du blanc. En fait ce n'est pas du beige mais plutôt une sorte de jaune maïs, vanille, des tons de jaune très doux. Avec du blanc. Et des meubles laqués blancs avec des planches en bois cérusées. Très distingué, tout ça. Et puis couleur cappuccino.

(Gabi, 51 ans, esthéticienne) 
appartement symbolise pour Gabi une stratégie à la fois de réussite et de distinction sociales. Réussite, car, après l'échec de son couple et le départ de son mari, elle s'était retrouvée, quelques années plus tôt, seule avec deux enfants, sans métier et sans ressources :

(n) Und wenn Sie bei mir mal in die Wohnung kommen, da sagen Sie : «Das gibt es nicht." Vom Harz 4-Empfänger, was ich mir aufgebaut hab. So ist noch keiner eingerichtet, der 15 Jahre berufstätig ist! Das ist für mich wichtig.
Et si un jour vous venez chez moi, dans mon appartement, vous direz: "Ce n'est pas possible." Ce que j'ai construit, en partant de l'allocation chômage minimum. Même quelqu'un qui travaille depuis quinze ans n'est pas aussi bien installé. C'est important pour moi.

Stratégie de distinction sociale aussi, car elle investit une part importante de son budget toujours relativement modeste (elle travaille en tant qu'esthéticienne salariée) dans son loyer. Habiter un appartement et un quartier qu'elle qualifie de « vernünftig » (correct) lui importe, au point qu'elle préfère renoncer à des vacances pour pouvoir payer un loyer élevé par rapport à ses moyens :

\begin{tabular}{|c|c|}
\hline $\begin{array}{l}\text { (o) Wie denn auch [in Urlaub fahren], als } \\
\text { Alleinerziehende? Ich hab... mir war immer } \\
\text { wichtig, 'ne vernünftige Wohnung, 'ne } \\
\text { vernünftige Umgebung. Und da bezahlst du } \\
\text { auch für. }\end{array}$ & $\begin{array}{l}\text { Comment j'aurais pu [partir en vacances], en } \\
\text { élevant seule mes enfants? J'ai... ce qui a toujours } \\
\text { été important pour moi, c'était un appartement } \\
\text { correct dans un environnement correct. Et ça a } \\
\text { son prix. }\end{array}$ \\
\hline
\end{tabular}

L'appartement est donc pour Gabi non seulement l'endroit où elle habite, mais aussi un espace qui lui permet d'afficher un "style», représentatif de son combat mené avec succès contre le déclin social dont elle avait été menacée. La couleur est alors un élément constitutif de ce style. Le lexique que Gabi déploie autour des couleurs n'appartient pas à la langue standard et fait preuve de notions certaines en matière de décoration: «Maisfarbe " (jaune maïs), "weiche Gelbtöne » (des tons de jaune doux), «Vanilla » (vanille), « cappuccinofarben » (couleur cappuccino). Ce sont des termes que l'on trouve typiquement dans des magazines spécialisés ou, comme le montre Dubois, dans des nuanciers de décoration:

29 "Dans les nuanciers de décoration, les formes lexicales utilisées renvoient massivement (dans plus de $50 \%$ des occurrences) à des sources, objets concrets dont la teinte est considérée typique pour la nuance (prune, jonquille, sable, liège...), bien que la véracité perceptive ne soit pas garantie (ex/ vanille pour un jaune), ou s'inscrit dans une référence plus abstraite mais symboliquement emblématique d'une couleur (océan, nuage, poterie, Sahara...). » (l'auteur souligne) ${ }^{24}$

Les termes qu'utilise Gabi correspondent au premier cas de figure qu'évoque Dubois: la couleur du maïs et de la vanille est considérée comme typique de jaunes clairs, pastel, celle du cappuccino est sans doute représentative d'un marron clair. Le fait que ce vocabulaire soit très probablement emprunté à un discours professionnel de décoration destiné au grand public devient particulièrement manifeste avec la désignation Vanilla qui, certes, ne désigne pas une couleur en allemand standard, mais qui, de surcroît, n'est 
pas un terme allemand tout court. En maitrisant cette terminologie, Gabi se présente non seulement comme une "experte" en matière de décoration, mais souligne encore davantage l'importance qu'elle accorde au choix des couleurs. Reste à constater que, dans notre échantillon, Gabi est la seule à parler de son appartement comme d'un espace dans lequel elle investit de l'énergie et de l'argent non pas prioritairement pour le rendre agréable à vivre, mais parce qu'elle voit dans cet investissement une stratégie de réussite sociale. Elle est en effet la seule à parler de son appartement comme d'un espace de représentation, dont la fonction première est d'être montré, d'être vu.

\section{L'habitat - une prolongation de soi-même}

Pour les autres informateurs, ce qui semble rendre le style de leur espace-logement difficilement désignable par un seul terme, par une étiquette, c'est aussi le rapport étroit, intime, qu'ils entretiennent avec cet espace. Nous avons déjà observé, à l'occasion des exemples cités plus haut, que le discours sur le chez soi mobilisait un lexique du bienêtre : " genießen » (jouir, profiter) (k), "sich wohlfühlen » (se sentir bien) (j), « Wärme » (chaleur) (h, i). Les extraits d'entretiens ci-dessous font encore mieux ressortir le rôle qu'attribuent les individus à leur habitat :

\begin{tabular}{|c|c|}
\hline $\begin{array}{l}\text { (p) Ich find's [= meine Wohnung] wichtig, also ich } \\
\text { wollte eigentlich auch 'ne, also 'ne } \\
\text { Eigentumswohnung haben, nicht zur Miete } \\
\text { wohnen, dass man irgendwo was Festes hat [...]. } \\
\text { Dass man zu Hause wohnt, irgendwie so was schon. } \\
\text { Nicht immer 'ne Mietwohnung. Die man auch so } \\
\text { einrichten kann, das ist auch schon schön. } \\
\text { (Joschi) } \\
\text { (q) Das Zuhause ist sehr wichtig. [...] ich denk mal, } \\
\text { das Zuhause, das ist so das Nest, das, also das steht } \\
\text { für mich doch schon vorne an. } \\
\text { (Meike) } \\
\text { (r) Häuslichkeit ist für mich wichtig, Häuslichkeit } \\
\text { bedeutet für mich mein persönliches Umfeld und } \\
\text { Geborgenheit. [...] Wohlfühlen. Geborgensein. } \\
\text { Eigenen Bereich zu haben. Ja, abgrenzen zu können. } \\
\text { (Annemarie) }\end{array}$ & $\begin{array}{l}\text { Je trouve que c'est [= mon appartement] } \\
\text { important, et au fond je voulais avoir un } \\
\text { logement qui m'appartient, ne pas être } \\
\text { locataire, avoir quelque chose de fixe quelque } \\
\text { part. [...] Habiter chez soi, d'une certaine } \\
\text { façon. Pas toujours dans un appartement en } \\
\text { location. Pouvoir l'agencer, ça aussi c'est } \\
\text { appréciable. } \\
\text { (Joschi) } \\
\text { Le chez-soi est très important. [...] je dirais } \\
\text { que le chez-soi, c'est comme un nid, enfin, } \\
\text { c'est une priorité pour moi. } \\
\text { (Meike) } \\
\text { Le foyer, c'est important pour moi, mon foyer } \\
\text { signifie pour moi mon environnement } \\
\text { personnel et un sentiment de sécurité. [...] Du } \\
\text { bien-être. Se sentir protégé. Avoir un espace à } \\
\text { soi. Oui, pouvoir se replier. } \\
\text { (Annemarie) }\end{array}$ \\
\hline
\end{tabular}

L'idée d'un espace qui lui appartient, qui constitue un point de repère fixe (" was Festes ») chez Joschi, la comparaison, chez Meike, du chez soi avec un nid (« das Nest»), et surtout les termes dans lesquels s'exprime Annemarie, pour qui la maison représente son environnement personnel (" persönliches Umfeld », « eigener Bereich»), un lieu protégé et de protection, un refuge ("Geborgenheit », «Geborgensein»), un lieu de bien-être («Wohlfühlen») et qui permet de se replier («abgrenzen») - tout ceci fait penser à l'habitat comme à une prolongation de soi-même. L'expression française du "chez soi », 
quand on la considère dans son sens littéral, en est, d'une certaine façon, révélatrice. La question "Wie sind Sie eingerichtet?» (Comment est votre intérieur? Comment c'est chez vous?) n'est d'ailleurs pas une question que l'on poserait à quelqu'un que l'on vient de rencontrer, ou que l'on connaît peu, car elle serait considérée - du moins dans nos aires culturelles - comme une violation de l'intimité de l'autre. L'intérieur de la maison ou de l'appartement de l'autre se découvre (et se commente généralement à cette occasion par des remarques élogieuses) parce qu'on est devenu suffisamment proche pour y être invité, mais sa description détaillée ne fait pas partie des sujets de conversation courants. Certains de nos informateurs, invités à parler de leur style d'intérieur lors d'une enquête qualitative, doivent donc aussi se sentir un peu pris de court, simplement parce qu'ils n'ont pas l'habitude que cette question leur soit adressée.

33 Abstraction faite de cette hypothèse, et pour ce qui est de la possibilité de parler de son style d'intérieur, l'analyse de notre corpus conduit aux constats - provisoires - suivants : premièrement, les locuteurs ne disposent pas (ou, en tout cas, ne mobilisent pas en parlant de leur propre logement) de termes permettant de désigner des styles d'intérieur. Deuxièmement, le style est décrit en comparaison avec d'autres styles dont il se démarque. Troisièmement, l'espace-logement est principalement abordé à travers des critères subjectifs : la description des ambiances que fournissent nos informateurs dans les extraits d'entretien cités ne permet pas à l'allocuté de se représenter concrètement l'espace, son ameublement et sa décoration ${ }^{25}$. Quatrièmement, l'espace-logement semble, pour certains locuteurs, s'appréhender plus naturellement par ce que l'on y fait que par ce que l'on y voit. À côté des éléments descriptifs (approche statique de l'espace) apparaissent alors des éléments narratifs (approche dynamique de l'espace).

Tout se passe comme si l'espace-logement était avant tout un espace que l'on expérimente, et quant au rapport entre le chez soi, indissociable de l'Homme, et l'activité langagière, il semble s'agir d'un espace qui se prête, comme toute expérience humaine, moins bien à être désigné ou décrit, et beaucoup mieux à être raconté.

\section{NOTES}

1. Norbert Wichard, 2012, Erzähltes Wohnen. Literarische Fortschreibungen eines Diskurskomplexes im bürgerlichen Zeitalter, Bielefeld, transcript Verlag, p. 18: «Habiter n'est pas seulement un processus élémentaire, indissociable de l'Homme, c'est également un concept qui “cherche" sa représentation langagière $[. .$.$] ».$

2. Nous nous inscrivons par là dans une approche sociologique, la sociologie des «styles de vie ", qui s'est développée au cours des années 80 , avec l'émergence de la thèse de l'individualisation. Suivant cette approche, dans les espaces de liberté dont dispose désormais l'individu postmoderne, les décisions qu'il prend, y compris les décisions de la vie quotidienne, seraient de moins en moins le fait de contraintes objectives, et de plus en plus le résultat de choix individuels et subjectifs. Sans nier que des déterminismes extérieurs continuent à exercer un impact sur l'organisation de la vie, la sociologie des styles de vie postule que le «moteur de l'action » se trouve désormais du côté de la subjectivité, et non pas du côté de ces données objectives extérieures. Pour un aperçu de l'histoire de cette approche, voir par exemple : Gunnar Otte, 2004, 
Sozialstrukturanalysen mit Lebensstilen, Eine Studie zur theoretischen und methodischen Neuorientierung der Lebensforschung, Wiesbaden, VS Verlag für Sozialwissenschaften.

3. Voir Britta Jabs, Styles de vie et façons de parler. Étude de quelques formes de discours dans différents milieux socio-culturels en Allemagne contemporaine, [inédit], thèse, Université Paris-Sorbonne, 2012.

4. Philippe Blanchet, 2000, La linguistique de terrain - Méthode et théorie, Rennes, Presses Universitaires de Rennes, p. 46-47.

5. Konrad Ehlich, 2007, "Alltägliches Erzählen», dans Konrad Ehlich (Hrsg.), Sprache und sprachliches Handeln, Band 3 : Diskurs - Narration - Text - Schrift, Berlin, New York, de Gruyter, p. 372-393.

6. Ehlich, 2007 (note 5), p. 373 : «Die Aufforderung, vom Urlaub zu erzählen, kann ebenso mit einem kurzen Bericht oder mit einer einfachen Darstellung des Urlaubsverlaufs beantwortet werden (“erzählen,") wie mit einer Reihe von kleinen Erzählungen (“erzählen ${ }_{2}$ "). »

7. Wolfgang Heinemann, Dieter Viehweger, 1991, Textlinguistik, Tübingen, Max Niemeyer Verlag, p. 282 : «Die Handlungen, die erzählt werden, müssen [...] für den Adressaten interessant sein, wobei ein Interesse in der Mehrzahl der Fälle dadurch erreicht wird, daß Abweichungen von den Normen festzustellen sind bzw. Erwartungen, die normalerweise im Verlauf einer Handlung eintreffen, sich nicht erfüllen. »

8. Jochen Rehbein, 1984, «Beschreiben, Berichten und Erzählen », dans Konrad Ehlich (Hrsg.), Erzählen in der Schule, Band 10: Kommunikation und Institution, Tübingen, Narr, p. 120: «[Es wird] erzählt, wie "es immer ist" ".

9. Rehbein, 1984 (note 8), p. 120.

10. Peter Auer, 1989, « Natürlichkeit und Stil », dans Volker Hinnenkamp, Margret Selting (éd.), Stil und Stilisierung, Tübingen, Max Niemeyer Verlag, p. 30 : «Oft sind [Stile] den Mitgliedern einer Kultur / Sprechgemeinschaft bewußt und teils sogar benennbar. Über Stil kann man sprechen [... ]. "

11. L'interview se déroule au domicile de Annemarie.

12. Concernant la technique d'interview, voir par exemple: Marita Roth, 2008, « Das narrative Interview als empirische Basis qualitativer Forschung ", dans Bernt Ahrenholz et al. (éd.), Empirische Forschung und Theoriebildung, Festschrift für Norbert Dittmar zum 65. Geburtstag, Frankfurt am Main, Peter Lang; Louis-Jean Calvet, Pierre Dumont (dir.), 1999, L'Enquête sociolinguistique, Paris, L'Harmattan; Susanne Uhmann, 1989, «Interviewstil : Konversationelle Eigenschaften eines sozialwissenschaftlichen Erhebungsinstruments.", dans Volker Hinnenkamp, Margret Selting (éd.), Stil und Stilisierung, Tübingen, Max Niemeyer Verlag, p. 125165.

13. On trouve toutefois une amorce chez Jürgen (c) qui emploie l'adjectif « kolonial »: en effet, « Kolonialstil » est un des termes utilisés par la presse spécialisée.

14. Rehbein, 1984 (note 8), p. 71 : «Beschreiben [ist] dem pragmatisch-funktionalen Bereich der räumlichen Orientierung zuzuordnen ».

15. Rehbein, 1984 (note 8), p. 69 : «beim Beschreiben macht der Hörer "einen Gang durch den Vorstellungsraum" ".

16. Rehbein, 1984 (note 8), p. 79 : «Durch die Richtung und die Stationen des Gangs wird ein Zusammenhang zwischen den einzelnen Elementen in der Vorstellung hergestellt. »

17. Rehbein (1984, note 8) envisage une acception assez large de la notion "spatiale» ( « räumlich») : « Der Sachverhalt beim Beschreiben hat zumeist den Charakter einer Räumlichkeit [...]. Werden Sachverhalte anderer Art beschrieben wie Werkzeuge, Bewegungen, Vorgehensweisen, Vorgänge, Verhaltensweisen usw., so dürfte es ihr jeweiliger räumlicher Aspekt sein, oder allgemeiner: ihr sichtbarer Aspekt, oder noch allgemeiner: ihr äußerer Aspekt, der wiedergegeben wird.» (p. 74 ; l'auteur souligne); trad. : «L'état de fait qui est l'objet d'une description se présente le plus souvent sous forme d'un espace [...]. Lorsqu'on décrit des états de fait d'un autre genre, comme par exemple des outils, des mouvements, des procédés, des procès, des façons de se comporter, ce 
qui est rendu, c'est sans doute leur aspect spatial, ou pour le dire de façon plus générale : leur aspect visible, ou de façon encore plus générale : leur aspect extérieur.»

18. $\mathrm{I}=$ Interviewer.

19. $E=$ Enquêteur.

20. Concernant l'histoire de la notion de style, et son appropriation par les sciences sociales, voir par exemple : Herbert Willems, 2003, "Stile, Stilgeneratoren und Stilfunktionen.", dans Stefan Habscheid, Ulla Fix (éd.), Gruppenstile, Forum Angewandte Linguistik, Band 42, Frankfurt am Main, Peter Lang, p. 15-30 ; Hans-Martin Gauger, 1995, « Was ist eigentlich Stil ? ", dans Gerhard Stickel (éd.), Stilfragen, Jahrbuch 1994 des Instituts für deutsche Sprache, Berlin, New York, Walter de Gruyter, p. 7-26; Wolf-Dieter Stempel, 1994, "Stylistique et interaction verbale.", dans Georges Molinié, Pierre Cahné (dir.), Qu'est-ce que le style ?, Paris, PUF, p. 313-330 ; Hans Ulrich Gumbrecht, K. Ludwig Pfeiffer (éd.), 1986, Stil -Geschichten und Funktionen eines kulturwissenschaftlichen Diskurselements, Frankfurt am Main, Suhrkamp Verlag; Elizabeht Closs Traugott, Suzanne Romaine, 1985, "Some questions for the definition of "style" in sociohistorical linguistics ", dans Folia linguistica historica, VI, p. 7-39.

21. Catherine Kerbrat-Orecchioni, 1998 (1986), L'Implicite, Paris, Armand Colin, p. 236.

22. Heinemann/Viehweger (note 7), p. 282: «Eine der grundlegenden Eigenschaften von konversationellen Erzählungen besteht darin, daß sich die Erzählinhalte auf Handlungen von Personen beziehen.»

23. Peter Berger, Thomas Luckmann, 1966 (trad. : 2003), La Construction sociale de la réalité, Paris, Armand Colin, p. 65. Voir aussi Gülich et Krafft qui parlent à ce propos de « savoir partagé d'un groupe » : Elisabeth Gülich, Ulrich Krafft, 2011, « Le Rôle du "préfabriqué" dans les processus de production discursive ", dans Michel Martins-Baltar (éd.), La Locution entre langue et usages, Lyon, ENS Editions, p. 255.

24. Danièle Dubois, 2001, «Catégorisation, langage et identité : Représentations individuelles et constructions symboliques partagées », dans Anne-Marie Costalat-Founeau (éd.), Identité sociale et langage : La Construction du sens, Collection Logiques Sociales, Paris, L'Harmattan, p. 210.

25. Les entretiens avec Mechthild, Helga, Annemarie et Jürgen se sont déroulés à leur domicile. Lorsque nous comparons les descriptions/caractérisations que ces informateurs livrent concernant leur style d'intérieur avec nos impressions personnelles, il s'avère que nous n'aurions guère employé les mêmes qualificatifs. Quant au style de Helga, nous le qualifierions de " gutbürgerlich» (bourgeois) (en écartant donc «modern» et «individuell»); quant au style de Annemarie, nous le qualifierions de «kitschig-überladen» (trop chargé et kitsch). Nous n'avons repéré aucune trace d'un élément que nous qualifierions de « südländisch » (méridional) ou de « kolonial» (colonial) dans la maison de Jürgen. Ceci semble être un indice supplémentaire confirmant que la perception des «styles » ne répond pas à des critères objectifs. - L'étonnement de Schulze, qui, décrivant l'intérieur idéaltypique du milieu qu'il identifie lui-même comme « Harmoniemilieu » (milieu à la recherche d'harmonie), constate qu'une autre étude emploie un tout autre vocabulaire, confirme cette impression : «Die in der Wohnwelt-Studie (1988, S. 70) abgebildeten milieuspezifischen Interieurs werden aus unerfindlichen Gründen als rustikal bezeichnet. Beherrschender Eindruck ist jedoch nicht Schlichtheit, sondern Anfüllung » (l'auteur souligne), trad. : «Les intérieurs typiques de ce milieu qui sont représentés dans la "Wohnwelt-Studie" (1988, p. 70) sont, pour des raisons incompréhensibles, qualifiés de "rustiques". L'impression dominante n'est pourtant pas celle de sobriété, mais celle d'encombrement." Voir Gerhard Schulze, 2005, Die Erlebnisgesellschaft. Kultursoziologie der Gegenwart, Frankfurt am Main, New York, Campus Verlag, p. 293. 


\section{ABSTRACTS}

Cette étude prend appui sur un corpus oral constitué par l'auteur à l'occasion d'une enquête qualitative menée en Allemagne, entre 2005 et 2010, et interroge plus particulièrement la façon dont les locuteurs parlent de leur style d'habitation. Elle confronte, à l'aide de nombreux extraits d'interview, trois formes de discours: «désigner ", "décrire» et « raconter ", en partant implicitement de l'idée que ses différentes activités langagières supposent chacune une plus ou moins grande distance objective, une plus ou moins forte implication affective de la part du locuteur. Les résultats de l'étude suggèrent que l'habitation (l'appartement, la maison), en tant qu'espace privé voire intime, est le plus aisément « racontée », c'est-à-dire appréhendée à travers une certaine forme de narration.

Die vorliegende Untersuchung greift auf ein mündliches Korpus zurück, das von der Autorin im Rahmen einer qualitiativen Feldforschung zwischen 2005 und 2010 in Deutschland erstellt wurde. Sie befasst sich insbesondere mit der Art und Weise, in der sich Sprecher über ihren Wohnstil äußern, und stellt diesbezüglich anhand zahlreicher Interviewausschnitte drei Diskursarten gegenüber : «bezeichnen», «beschreiben» und "erzählen». Dabei wird implizit davon ausgegangen, dass diese unterschiedlichen Formen sprachlichen Handelns jeweils eine mehr oder weniger große objektive Distanz bzw. eine mehr oder weniger starke emotionale Einbindung des Sprechers voraussetzen. Tatsächlich lassen die Ergebnisse der Untersuchung vermuten, dass es Sprechern am leichtesten fällt, sich über den privaten, intimen Raum (ihre Wohnung oder ihr Haus) in einer bestimmten Form von Erzählungen mitzuteilen.

\section{INDEX}

Chronological index: XXIe siècle

Geographical index: Allemagne

Mots-clés: analyse de discours, enquête qualitative, espace privé, habitat, style de vie

\section{AUTHOR}

\section{BRITTA JALLERAT-JABS}

Britta Jallerat-Jabs, agrégée d'allemand et docteur en linguistique germanique, est maître de conférences à l'Université Paris 3 - Sorbonne Nouvelle, où elle enseigne notamment la sociolinguistique, la grammaire de l'allemand contemporain et la traduction français-allemand. Elle y assure également la responsabilité du Master MEEF (Métiers de l'Enseignement, de l'Education et de la Formation) allemand, et elle est membre du jury de l'agrégation externe d'allemand depuis 2014. Ses recherches portent principalement sur les formes de discours dans différents milieux socioculturels en Allemagne contemporaine. 